\title{
The effect of high-speed dental handpiece coolant delivery and design on aerosol and droplet production
}

Short title: Aerosol from high-speed dental handpieces

\author{
James R Allison ${ }^{\text {a, }}$; Clinical Fellow in Oral Surgery \\ David Edwards a, b; NIHR Academic Clinical Fellow in Endodontics \\ Charlotte Bowes ${ }^{a}$; ; NIHR In-Practice Fellow \\ Kimberley Pickering ${ }^{\text {b; }}$ Senior Research Dental Nurse \\ Christopher Dowson ${ }^{a}$; PhD Candidate \\ Simon J Stone ${ }^{a, b}$; Senior Clinical Lecturer, Honorary Consultant in Endodontics \\ Joanna Lumb b; Consultant Microbiologist \\ Justin Durham ${ }^{\mathrm{a}, \mathrm{b}}$; Head of School, Professor of Orofacial Pain, Honorary Consultant Oral \\ Surgeon \\ Nicholas Jakubovics ${ }^{\text {a }}$; Senior Lecturer in Oral Microbiology \\ Richard Holliday ${ }^{a, b}$; Senior Clinical Lecturer and Honorary Consultant in Restorative \\ Dentistry
}

\section{Affiliations:}

a. School of Dental Sciences, Faculty of Medical Sciences, Newcastle University, United Kingdom

b. Newcastle upon Tyne Hospitals NHS Foundation Trust, Newcastle Upon Tyne, United Kingdom

* Corresponding Author:

Dr Richard Holliday

School of Dental Sciences

Faculty of Medical Sciences

Newcastle University

Framlington Place

Newcastle Upon Tyne

NE2 4BW

07876580022

Richard.holliday@newcastle.ac.uk 
Keywords: Bioaerosols; Aerosol Generating Procedures; Dental Equipment; Dental Infection Control; SARS-CoV-2; 
Objectives: High-speed dental instruments produce aerosol and droplets. The objective of this study was to evaluate aerosol and droplet production from a novel electric micromotor handpiece (without compressed air coolant) in real world clinical settings.

Methods: 10-minute upper incisor crown preparations were performed in triplicate in an open-plan clinic with mechanical ventilation providing 3.45 air changes per hour. A 1:5 ratio electric micromotor handpiece which allows water coolant without compressed air (Ti-Max Z95L, NSK) was used at three speeds: 60,000 (60K), 120,000 (120K), and 200,000 (200K) revolutions per minute. Coolant solutions contained fluorescein sodium as a tracer $(2.65$ $\left.\mathrm{mmol} \mathrm{L}^{-1}\right)$. High-speed air-turbine positive control, and negative control conditions were conducted. Aerosol production was evaluated at 3 locations $(0.5 \mathrm{~m}, 1.5 \mathrm{~m}$ and $1.7 \mathrm{~m})$ using: (1) an optical particle counter (OPC; 3016-IAQ, Lighthouse) to detect all aerosol; and (2) a liquid cyclone air sampler (BioSampler, SKC Ltd.) to detect aerosolised fluorescein, which was quantified by spectrofluorometric analysis. Settled droplets were detected by spectrofluorometric analysis of filter papers placed onto a rig across the open-plan clinic.

Results: Local (within treatment bay) settled droplet contamination was elevated above negative control for all conditions, with no difference between conditions. Settled droplet contamination was not detected above negative controls outside the treatment bay for any condition. Aerosol detection at $1.5 \mathrm{~m}$ and $1.7 \mathrm{~m}$, was only increased for the air-turbine positive control condition. At $0.5 \mathrm{~m}$, aerosol levels were highly elevated for the air-turbine, minimally elevated for $200 \mathrm{~K}$ and $120 \mathrm{~K}$, and not elevated for $60 \mathrm{~K}$.

Conclusions: Electric micromotor handpieces which use water-jet coolant alone without compressed air, produce localised (within treatment bay) droplet contamination but are unlikely to produce aerosol contamination beyond the immediate treatment area $(1.5 \mathrm{~m})$, allowing them to be used safely in most open-plan clinic settings. 


\section{Introduction}

Dental procedures performed using high-speed instruments produce aerosol and droplets contaminated with saliva and blood (Miller 1995, Bennett et al. 2000); transmission of oral pathogens is therefore possible. The coronavirus disease 2019 (COVID-19) pandemic has had a significant impact on dental services due to concerns over dispersion of severe acute respiratory syndrome coronavirus 2 (SARS-CoV-2) from the mouth in aerosols and droplets produced by dental instruments. One study of hospitalised patients reported mean salivary SARS-CoV-2 RNA concentration of $3.80 \times 10^{5}\left(95 \% \mathrm{Cl}: 1.23 \times 10^{5}-1.17 \times 10^{6}\right)$ copies $/ \mathrm{mL}$, with lower levels in a group of asymptomatic individuals (Wyllie et al. 2020), and SARS-CoV-2 RNA has also been isolated from gingival crevicular fluid (Gupta et al. 2020). Data suggest that SARS-CoV-2 may remain infectious in aerosols (van Doremalen et al. 2020), although the number of virions potentially carried in aerosols from dental procedures is unknown. Although there is likely to be a dilution effect (Holliday et al. 2021), the duration of dental procedures and proximity of practitioners to the source leave potential for significant exposure. Data demonstrate that dental suction and ventilation have a significant beneficial effect on the amount of contamination escaping the immediate locality of the procedure (Allison et al. 2021, Holliday et al. 2021), however the amount of contamination produced by different procedures is likely to vary.

High-speed dental instruments are used in many applications within the practice of dentistry, for example when ablating tooth tissue during restorative procedures, during the removal and polishing of restorative materials, when removing bone during surgical procedures, and when adjusting dental appliances. There are generally two ways of transferring rotational force to burs used for dental procedures. The first is with an air- 
turbine handpiece which uses compressed air to drive a turbine rotor housed within the head of the handpiece, and these devices operate at speeds up to 400,000 revolutions per minute (rpm). The second is by using a geared handpiece, which are available in several gear ratios (e.g. 10:1, 1:1, 1:4.2, 1:5) and are commonly referred to as "slow-speed", "speedincreasing", "straight", or "contra-angle" handpieces. Geared handpieces can be driven either by a dental air-motor, which uses a low-torque rotary vane motor driven by compressed air at output speeds up to $25,000 \mathrm{rpm}$, or an electric micromotor with output speeds up to $40,000 \mathrm{rpm}$. With a 1:5 ratio geared handpiece bur speeds of up to 200,000 rpm are therefore possible. An additional benefit of electric micromotor driven handpieces is that high torque can be maintained when the bur is under higher loads (e.g., when cutting tooth tissue) unlike with compressed air systems. Heat produced by ablation using these instruments is managed by directing water via the handpiece to the operative site. Airturbine handpieces typically premix coolant water with compressed air (so called "chip-air") before directing this onto the operative site, thus producing a pre-misted coolant spray containing a mixture of large and small droplets and aerosol. Geared handpieces driven by air-motor and electric micromotors often use a similar coolant arrangement and due to turbulent mixing of water with air, the droplets produced can have high velocities, as demonstrated in a recent study examining these droplet's close-field dynamics (Sergis et al. 2020). Droplets produced by instruments using water and compressed air cooling may therefore be deposited at distant sites (Allison et al. 2021, Holliday et al. 2021). The potential mixing of coolant with saliva and blood prior to dispersion poses a risk of transmitting salivary pathogens (such as SARS-CoV-2). Some handpieces do not premix compressed air with coolant water, instead directing a steady stream of water alone onto the bur. It is unclear whether using jets of coolant water in high-speed dental instruments 
without the use of compressed air makes a difference to the pattern or distribution of potential contamination produced.

The aim of the present study was to evaluate aerosol and droplet contamination across a real-world clinical area from the use of geared handpiece driven by an electric micromotor with water-jet coolant (i.e., water alone without compressed air), compared to a high-speed air turbine handpiece.

\section{Materials and Methods}

\section{Clinical setting}

Experiments were conducted in an open plan clinic of a dental teaching hospital with a supply and extract mechanical ventilation system providing 3.45 air changes per hour $(\mathrm{ACH}$; this was assessed by an external engineering contractor). A schematic of the ventilation configuration is included in Figure 2. Half of a $603 \mathrm{~m}^{3}$, 21-bay chair clinic was used, and this setting is described in detail in our previous work (Holliday et al. 2021). During the experiments, only the operator and assistant were present inside the experimental area and left immediately after completing the dental procedure.

\section{Dental procedures}

A dental mannequin (P-6/3 TSE, Frasaco GmbH; Tettnang, Germany) was attached to a dental chair (Pelton and Crane Spirit Series, Charlotte, USA) and the mouth of the mannequin was positioned $73 \mathrm{~cm}$ above the floor. One of two operators ( $\mathrm{RH}$, height: 170 $\mathrm{cm}$; DE, height: $187 \mathrm{~cm}$ ) completed a crown preparation on the right maxillary central incisor tooth (AG-3, Frasaco GmbH; Tettnang, Germany) for a duration of ten minutes. An assistant held dental suction with an $8.2 \mathrm{~mm}$ diameter tip at a flow rate of $159 \mathrm{~L} / \mathrm{min}$ air 
measured using a flow meter (Ramvac Flowcheck, DentalEZ; PA, USA); this equates to "medium volume suction" according to UK national HTM 2022 guidelines (NHS Estates 2003). The procedure was performed using diamond burs (Rugby Ball 379, Shoulder 85656 \& 856G, Drendel and Zweiling, Germany) in either an air-rotor handpiece (Synea, W\&H, Bürmoos, Austria), or a 1:5 speed increasing handpiece (Ti-Max Z95L, NSK; Tochigi, Japan) driven by an electric micromotor (NLX Nano, NSK; Tochigi, Japan), further referred to as 'micromotor' (coolant flow rate of $14 \mathrm{~mL} / \mathrm{min}$ ). The micromotor had two coolant settings: coolant water mixed with compressed air (further termed 'spray') and coolant water alone (further termed 'water-jet'; Figure 1). In the preliminary studies (described below) both settings were evaluated, but for the definitive experiments in the clinical setting only the water-jet setting was used. Table 1 summarises the experimental parameters tested. Three replicates were conducted for each experiment. A negative control condition was completed five times.

\section{Table 1}

\begin{tabular}{|c|c|c|c|}
\hline Handpiece design & Speed (rpm) & Coolant delivery & Abbreviations \\
\hline High-speed air-turbine & $\begin{array}{l}\text { Approx. } \\
400,000\end{array}$ & $\begin{array}{c}\text { Water mixed with } \\
\text { compressed air }\end{array}$ & Air-turbine \\
\hline \multirow{3}{*}{ Electric micromotor } & 60,000 & \multirow{3}{*}{ Water-jet } & Micromotor 60K \\
\hline & 120,000 & & Micromotor $120 \mathrm{~K}$ \\
\hline & 200,000 & & Micromotor 200K \\
\hline \multicolumn{2}{|c|}{ Negative control } & $\mathrm{N} / \mathrm{A}$ & Control \\
\hline
\end{tabular}




\section{Aerosol and droplet detection}

Three complementary methods were used to assess the contamination across a clinical area: spectrofluorometric analysis of filters papers (assessing droplets and settled aerosols), active sampling with an optical particle counter (non-specific measurement of suspended aerosols), and active sampling with an air sampler and spectrofluorometric analysis of collection liquid (measurement of suspended fluorescein-containing aerosols). Each of the detection methods were completed in triplicate at three locations: $0.5 \mathrm{~m}$ (patients' chest), $1.5 \mathrm{~m}$ (bench), $1.7 \mathrm{~m}$ (walkway). Figure 2 illustrates these sampling positions.

The method for assessing droplets and settled aerosols has been previously reported in detail (Allison et al. 2021, Holliday et al. 2021, Llandro et al. 2021). In summary, 70 filter papers (30 mm diameter grade 1 qualitative cotton-cellulose; Whatman, Cytiva; MA, USA) were positioned on clean platforms across the experimental area. These were left in place during the procedure and for 30 minutes after, before being collected and analysed by eluting fluorescein from filter papers using distilled water. Fluorescence of was then measured for each sample using spectrofluorometry to give a reading in relative fluorescence units (RFU) equating to the amount of fluorescein tracer captured on each filter paper.

A laser-diode optical particle counter (OPC; 3016 IAQ, Lighthouse Worldwide Solutions; OR, USA) was used to measure suspended aerosols. The OPC had six particle-size channels $(0.3$, $0.5,1.0,2.5,5.0$, and $10.0 \mu \mathrm{m}$ ) with a sampling flow rate of $2.83 \mathrm{~L} / \mathrm{min}$ and was calibrated by the manufacturer to ISO 21501-4 standards. The instrument was set to sample continuously for 5 second intervals, beginning 1 minute before the procedure, and 
continuing during the procedure and for 30 minutes after. The OPC was placed in one of three positions during each experiment $(0.5 \mathrm{~m}, 1.5 \mathrm{~m}, 1.7 \mathrm{~m}$; Figure 2). Data were presented as normalised particle counts (particles/ $\mathrm{m}^{3}$ ) over the time-course of the experiment and total particle counts were summed across all channels. As experiments were conducted in real clinical settings, background particle counts were highly variable. All OPC data were therefore normalised to an internal baseline by subtracting the average counts during the 1 minute before the procedure from all particle counts. This instrument was also used to measure temperature and relative humidity at the same sampling intervals.

Liquid cyclone air samplers (BioSampler, SKC Inc.; PA, USA) were cleaned using alternating washing with distilled water and $70 \%$ ethanol to eliminate carry-over of fluorescein, which was validated by preliminary testing. A BioSampler was placed in one of three positions during each experiment $(0.5 \mathrm{~m}, 1.5 \mathrm{~m}, 1.7 \mathrm{~m})$ and $20 \mathrm{~mL}$ of distilled water was added to the sampling vessel. The device was operated at $12.5 \mathrm{~L} / \mathrm{min}$ using a sampling pump (BioLite+, SKC Inc.; PA, USA) and calibrated using a rotameter (SKC Inc.; PA, USA). Sampling began at 1 minute before the 10-minute dental procedure and continued until 30 minutes after the end of the procedure (41 minutes in total).

\section{Preliminary experiments}

Preliminary experiments were conducted to evaluate the two coolant settings of the handpiece (spray and water-jet). The methods for this preliminary work are described in detail in the Supporting Information (available online). 


\section{$\underline{\text { Statistics }}$}

Data were collected using Excel (2016, Microsoft; WA, USA) and analysed using SPSS (version24, IBM Corp.; NY, USA) using descriptive statistics.

\section{Results}

Full datasets from this project are publicly available

(https://doi.org/10.25405/data.ncl.14518950). During experiments in the clinical setting, the mean (SD; minimum - maximum) temperature was $21.9^{\circ} \mathrm{C}\left(0.9 ; 17.5-23.2^{\circ} \mathrm{C}\right)$ and relative humidity was $28.7 \%(4.9 ; 16.9-41.9)$.

\section{Preliminary experiments}

Data from preliminary experiments, conducted in a laboratory setting, demonstrated that there was a considerable difference between the two micromotor handpiece coolant conditions (spray and water-jet). Within the first $1 \mathrm{~m}$, both coolant settings produced large amounts of settled droplet contamination (pooling data from the three repetitions; spray: 705,160 RFU, water-jet: 2,133,619 RFU). Beyond 1m (i.e., between $1.5-4 \mathrm{~m}$ ) there was a 11.5-fold reduction in settled droplet contamination for the water-jet setting compared to the spray setting (pooling data from the three repetitions; spray: $28,980 \mathrm{RFU}$, water-jet: 2,523 RFU). Detailed results including heatmaps for the two conditions are presented in the Supporting Information (available online). 
$\underline{\text { Passive sampling with spectrofluorometric analysis of filter papers (settled droplets) }}$

1,190 filter paper samples were collected across the five experimental conditions (Air-

turbine, micromotor $60 \mathrm{~K}$, micromotor $120 \mathrm{~K}$, micromotor $200 \mathrm{~K}$, control). For each condition the data were divided into categories: samples collected from within the bay in which the procedure was performed (AGP bay) or its associated walkway; and samples collected from the rest of the clinic (Figure 3). Within the AGP bay/walkway the micromotor handpiece at all speeds produced comparable settled droplet contamination to the air-turbine. Outside of the AGP bay/walkway none of the conditions produced settled droplet contamination above the lower threshold of detection (mean of control +3 SD).

\section{Active sampling with an optical particle counter (suspended aerosols)}

Data from the OPC, representing a sensitive and non-specific method of detecting suspended particles between $0.3-10.0 \mu \mathrm{m}$, demonstrated that the air-turbine produced several peaks in particle counts at all three sampling locations, although these reduced in magnitude with increasing distance from the source. At $0.5 \mathrm{~m}$ the particle count was raised throughout the procedure and reduced to baseline levels within 6 minutes. The micromotor handpiece showed some elevation in particle counts at $0.5 \mathrm{~m}$ at all speeds, although this was considerably lower than the air-turbine; no elevation was seen at $1.5 \mathrm{~m}$ and $1.7 \mathrm{~m}$ for the micromotor handpiece at any speed. Figure 4 shows representative particle count-timeseries data for one repetition. All three repetitions in each location showed a similar pattern, and these data available on the Supporting Information (Figure S3-5, available online). 


\section{Active sampling with BioSampler and spectrofluorometric analysis}

Figure 5 shows the result of active sampling of aerosolised fluorescein tracer using the BioSampler. High readings were observed with the air-turbine condition at all three locations, indicting a large amount of suspended fluorescein-containing droplets. The micromotor produced levels below the lower threshold of detection (mean of control +3 $\mathrm{SD})$ at the 1.5 and $1.7 \mathrm{~m}$ sampling positions at all speed settings. At the $0.5 \mathrm{~m}$ sampling position, aerosolised fluorescein was detectable just above the lower threshold of detection for the micromotor at $120 \mathrm{~K}$ and $200 \mathrm{~K}$, however this was an order of magnitude lower than the air-turbine reading.

\section{Discussion}

Overall, three complementary sets of data from multiple sampling locations across multiple replicates robustly demonstrate that the electric micromotor handpiece with water-jet coolant produces localised (within the treatment bay) settled droplet contamination but is unlikely to produce aerosol contamination beyond the immediate treatment area $(1.5 \mathrm{~m})$ at any speed setting. In the close-field (i.e., within $0.5 \mathrm{~m}$ ) aerosol is produced when higher speeds are used $(120,000$ and $200,000 \mathrm{rpm})$, although the amount produced is much lower than with an air-turbine.

A recent study (Sergis et al. 2020) investigated the close-field characterisation of aerosols and droplets production by electric micromotor handpieces with conventional air/water spray and water-jet coolant systems and at a range of speeds. Using high speed imaging over the first $0.2 \mathrm{~m}$ from the procedure, the authors demonstrated that significantly less aerosol is produced when air and water are not pre-misted (as with water-jet irrigation), 
and that droplets are likely to be larger and travel shorter distances from the source. The authors also concluded that aerosol production by contact of irrigant with a spinning bur is reduced at bur speeds below 100,000 rpm. OPC data presented by Sergis et al. demonstrated that a micromotor handpiece (with the same parameters as used in our study) produced considerably lower particle counts than the air-turbine at $1.5 \mathrm{~m}$. Slight particle count increases above baseline were only seen with the micromotor handpiece at 150,000 and $200,000 \mathrm{rpm}$. This is in keeping with our real-world experiments in which we saw a similar pattern at our closest sampling location $(0.5 \mathrm{~m})$, however, we demonstrate with additional methods, that aerosol and droplet contamination is not significant at micromotor handpiece bur speeds up to $200,000 \mathrm{rpm}$ at $1.5 \mathrm{~m}$. OPC data can be hard to interpret due to the sensitive and non-specific nature of this measurement-elevated particle counts may be unrelated to the parameter under investigation. This is particularly true in real-world settings where background particle counts can be high (Thompkins et al. 2020), and is supported by the conclusions of another recent study which combined OPC data with a viral tracer (Vernon et al. 2021). By using three complementary analysis methods (active and passive tracer sampling, and OPC) in the present study, we are able provide high confidence in our findings.

Current UK guidance (Public Health England 2020) suggests that the use of rotary instruments below $60,000 \mathrm{rpm}$ leads to minimal aerosol production. Interestingly, these recommendations based on the work of (Sergis et al. 2020) do not mention the type of coolant setting used (i.e., water-jet alone or combined with compressed air) a parameter which this paper and now the present study has shown is an important consideration. Future studies and guidance should clearly state the coolant delivery system used. This 
guidance also recommends fallow-time (during which dental surgeries should be left unused) following dental procedures of up to 30-minutes. Our OPC data demonstrate that particle counts decay rapidly, even when an air-turbine handpiece is used, and in a setting with low to moderate ventilation rate $(3.45 \mathrm{ACH})$. Although OPC data has limitations as discussed, this nonetheless suggests that such fallow-time recommendations may be overcautious (particularly when utilising multiple mitigation strategies: dental suction, rubber dam, and pre-screening of patients).

The authors found that conducting operative dentistry using a micromotor handpiece at reduced bur speeds (e.g., 60,000 - 80,000 rpm) was challenging for anything more than minimal adjustments and these speeds are likely to be inappropriate for novice users. We found working at 120,000 rpm (or above) acceptable and hypothesise that these higher speeds have improved cutting efficiency and may cause less thermal change in the pulp. These are however simply the observations of the investigators and quantitative assessment was not undertaken.

The present study has several limitations. The study was conducted on a mannequin rather than in patients and respiratory activities such as breathing, talking, or coughing were therefore not modelled; however, the study aimed to understand the additional risk that dental procedures produce over and above normal clinical contact, and an experimental design using a mannequin allows systematic investigation of this in a controlled way. The use of a fluorescent tracer is a reasonably straightforward approach to examine the distribution of dental aerosols, however the biological characteristics of bioaerosols cannot be examined, such as the infectivity of any dispersed viruses. Future studies should utilise a 
biological tracer to validate the findings from non-biological models such as those used in the present study.

Our findings build on previous data and support the conclusion that micromotor handpieces with water-jet coolant system produce minimal aerosol and that any aerosol produced is distributed in the immediate vicinity of the procedure. Our findings demonstrate that this remains the case at higher bur speeds outside $1.5 \mathrm{~m}$. This has implications for open plan clinical environments and indicates that micromotor handpieces with water-jet irrigation can be used at the full range of speed setting provided those in the immediate vicinity (1.5m) use appropriate respiratory (FFP3), eye (visor), and clothing protection.

\section{Conclusion}

Combining the findings from three analysis techniques capturing suspended aerosols and settled droplets, our data demonstrate that electric micromotor handpieces with water-jet coolant produce considerably lower levels of contamination compared to high-speed airturbine handpieces. Localised (within treatment bay) droplet contamination was present and was similar to when an air-turbine handpiece was used. No contaminated aerosol was detected beyond the immediate treatment area $(1.5 \mathrm{~m})$ at any speed setting $(60,000-$ $200,000 \mathrm{rpm}$ ) indicating the improved safety profile of this design for open-plan clinic settings. At higher bur speeds, aerosols are produced in the immediate vicinity of the procedure, hence suitable respiratory protection should be used by those involved in the procedure. 


\section{Figure Legends}

Figure 1. Spray (a) and water-jet coolant (b) systems on an electric micromotor handpiece. Figure 2. Plan view of the experimental area showing sampling positions at $0.5,1.5$ and 1.7 $\mathrm{m}$. Red star indicates the location of the aerosol generating procedure. Air intake (square vents) and air output (long vents) are shown. (For interpretation of the references to colour in this figure legend, the reader is referred to the web version of this article). This figure is modified from Holliday et al.

Figure 3. Settled droplet contamination measured by spectrofluorimetric analysis of filter paper samples. Samples divided into two categories: Aerosol Generating Procedure (AGP) bay/walkway and outside of the AGP bay/walkway. A lower threshold of detection is provided for each category based upon the control condition + 3SDs. Error bars represent two SEM. RFU: Relative Fluorescence Units.

Figure 4. Suspended droplets as measured by an optical particle counter. Illustrative traces given in this figure from one repetition, data from all repetitions available in the Supporting Information (available online). Data collected from three sampling locations: (a) $0.5 \mathrm{~m}$, (b) $1.5 \mathrm{~m}$, (c) $1.7 \mathrm{~m}$. Data were presented as normalised particle counts (particles/ $\mathrm{m}^{3}$ ) over the time-course of the experiment and total particle counts were summed across all channels. All OPC data were normalised to an internal baseline by subtracting the average counts during the 1 minute before the procedure from all particle counts.

Figure 5. Suspended fluorescein-containing droplets as measured by BioSampler collection and spectroflurometric analysis. A lower level of detection is provided for each category based upon the control condition + 3SDs. RFU: Relative Fluorescence Units. 


\section{References}

Allison JR, Currie CC, Edwards DC et al. (2021) Evaluating aerosol and splatter following dental procedures: addressing new challenges for oral healthcare and rehabilitation. Journal of Oral Rehabilitation 48, 61-72.

Bennett AM, Fulford MR, Walker JT, Bradshaw DJ, Martin MV, Marsh PD (2000) Microbial aerosols in general dental practice. British Dental Journal 189, 664-667.

Gupta S, Mohindra R, Chauhan PK et al. (2020) SARS-CoV-2 Detection in Gingival Crevicular Fluid. J Dent Res, 22034520970536.

Holliday R, Allison J, Currie C et al. (2021) Evaluating contaminated dental aerosol and splatter in an open plan clinic environment: Implications for the COVID-19 pandemic. J Dent 105, DOI:10.1016/j.jdent.2020.103565.

Llandro H, Allison J, Currie C et al. (2021) Evaluating splatter and settled aerosol during orthodontic debonding: implications for the COVID-19 pandemic. Br Dent J 230, DOI:10.1038/s41415-41020-42503-41419.

Miller RL (1995) Characteristics of Blood-Containing Aerosols Generated by Common Powered Dental Instruments. American Industrial Hygiene Association Journal 56, 670-676.

NHS Estates (2003) HTM 2022 - Supplement 1: Dental compressed air and vacuum systems. London: The Stationary Office.

Public Health England (2020) COVID-19: infection prevention and control dental appendix. London: Public Health England.

Sergis A, Wade W, Gallagher J et al. (2020) Mechanisms of atomization from rotary dental instruments and its mitigation. Journal of Dental Research DOI: 10.1177/0022034520979644.

Thompkins J, Quincey P, Allerton J, Williams K (2020) Feasibility study on the detection of airborne particulates in a dental surgery using an optical particle counter. Middlesex, UK: National Physical Laboratory.

van Doremalen N, Bushmaker T, Morris DH et al. (2020) Aerosol and Surface Stability of SARS-CoV-2 as Compared with SARS-CoV-1. NEJM 382, 1564-1567.

Vernon JJ, Black EVI, Dennis T et al. (2021) Dental mitigation strategies to reduce aerosolization of SARS-CoV-2. medRxiv, 2021.2003.2024.21254254.

Wyllie AL, Fournier J, Casanovas-Massana A et al. (2020) Saliva or Nasopharyngeal Swab Specimens for Detection of SARS-CoV-2. The New England journal of medicine 383, 1283-1286. 
(a)

(b)

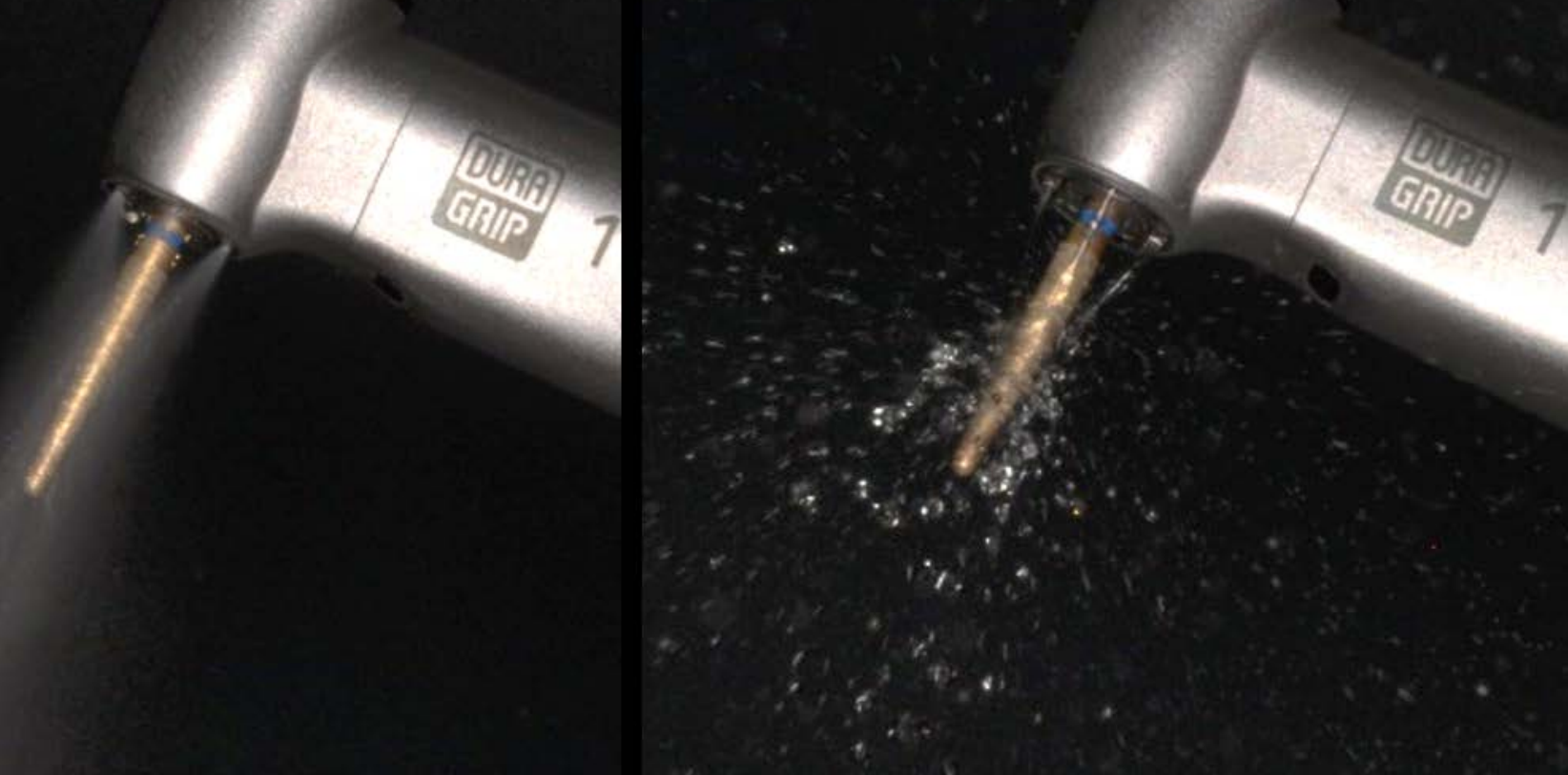



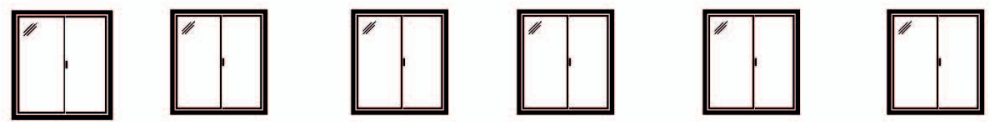

$16 \mathrm{~m}$

$7 \mathrm{~m}$

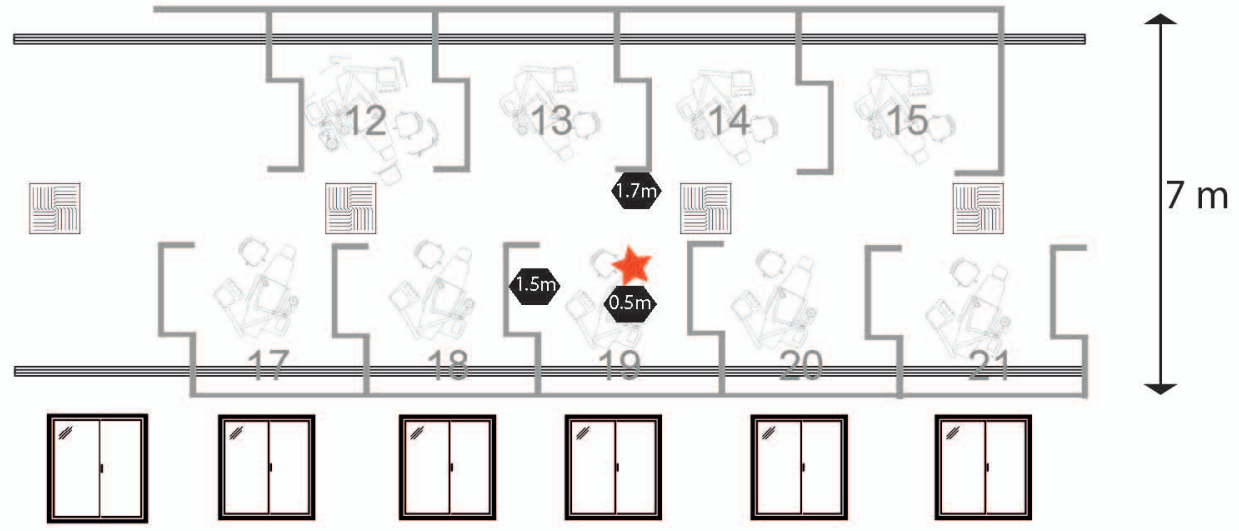




\section{0}

160000

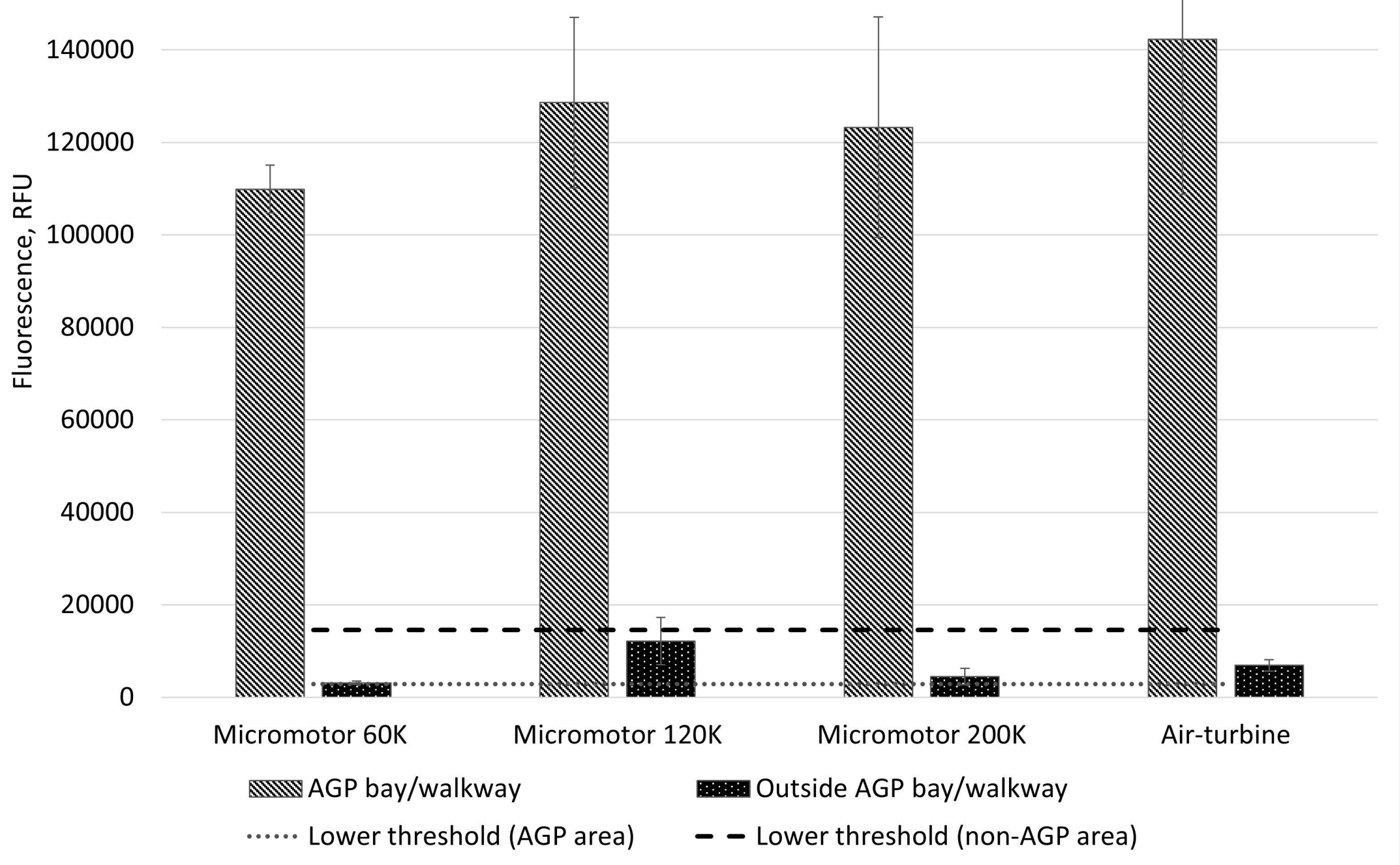




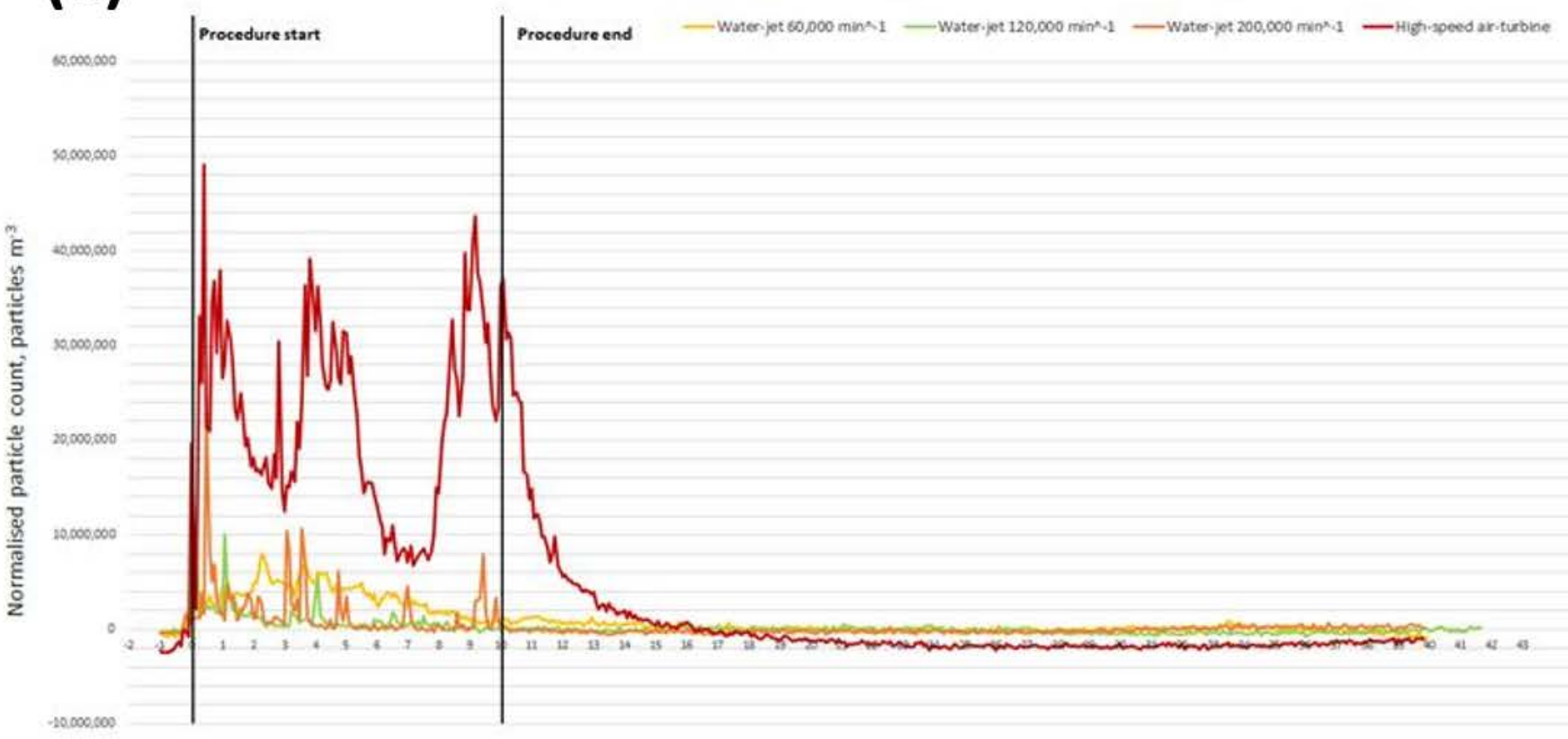

Time, minutes

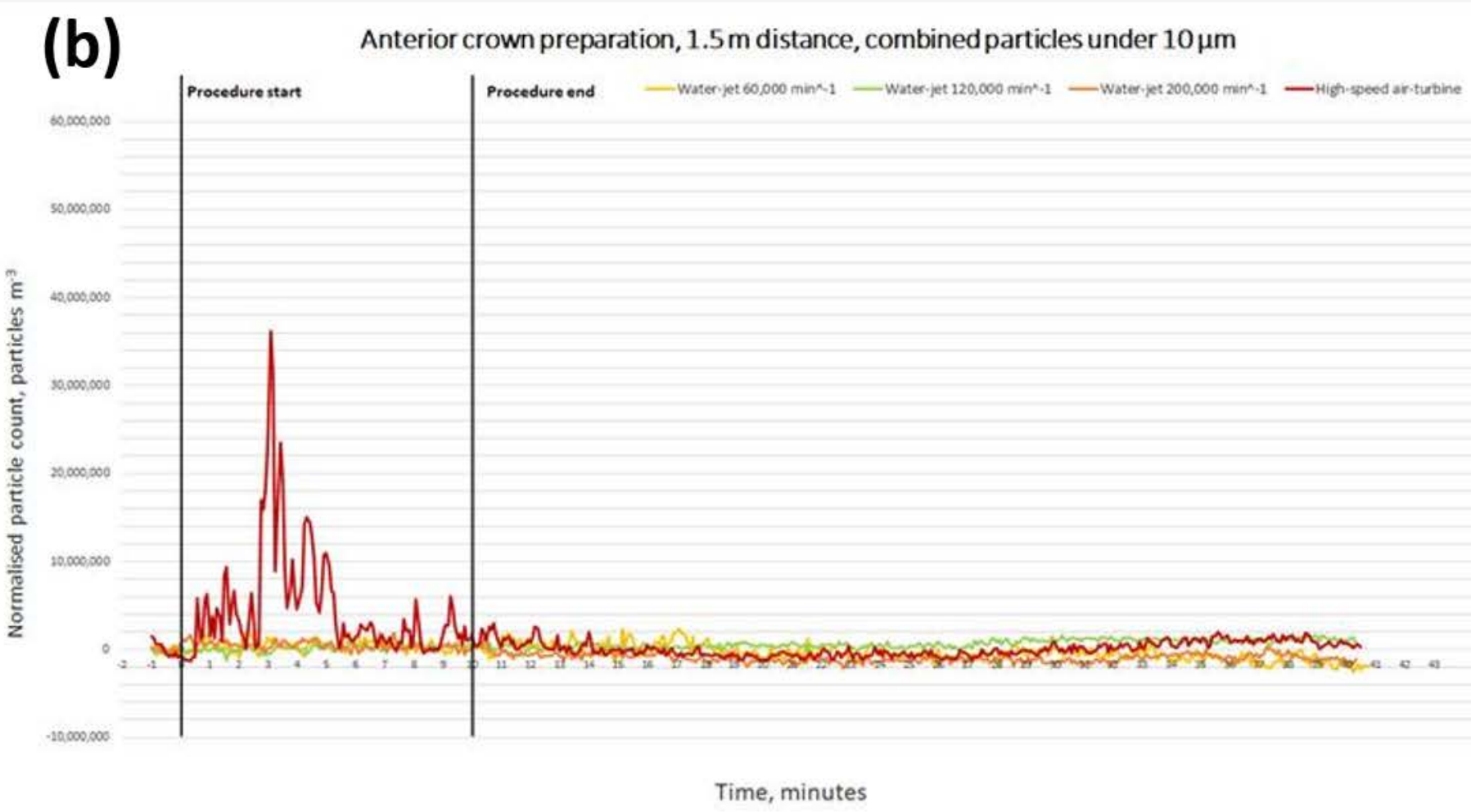

(c)

Anterior crown preparation, $1.7 \mathrm{~m}$ distance, combined particles under $10 \mu \mathrm{m}$

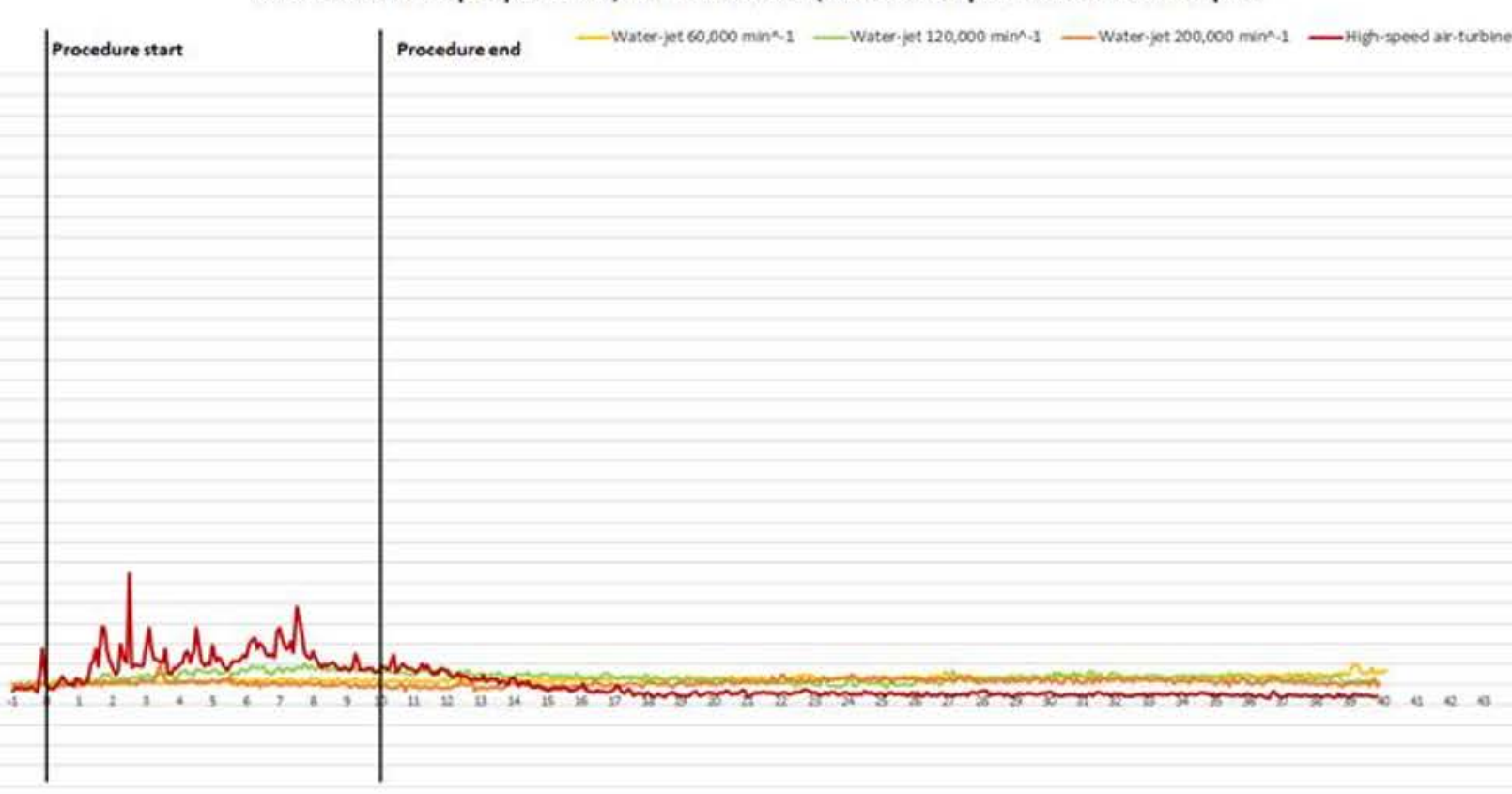


1600

1400

1200

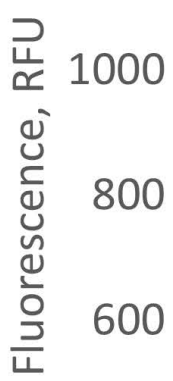

400

200

0

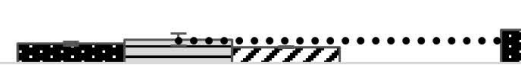

Micromotor 60K
Micromotor $120 \mathrm{~K}$

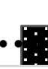

Micromotor 200K

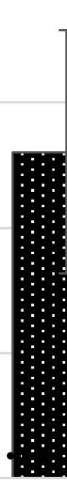

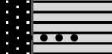

Air-turbine

0.5 m (mannequin chest) 


\section{Acknowledgements}

This work was supported by research grants from the British Endodontic Society and the Royal College of Surgeons of Edinburgh, and by the School of Dental Sciences, Newcastle University. Richard Holliday was funded by a National Institute for Health Research (NIHR) Clinical Lectureship. David Edwards is funded by an NIHR Academic Clinical Fellowship. Charlotte Bowes is funded by an NIHR In-Practice Fellowship. Christopher Dowson is funded by an NIHR Newcastle Biomedical Research Centre Studentship. The views expressed are those of the authors and not necessarily those of the National Health Service, NIHR or Department of Health and Social Care. We would like to thank Ekaterina Kozhevnikova and Dr Christopher Nile for their wider support for this work. Handpieces were provided on-loan to Newcastle Dental Hospital for local evaluation by NSK United Kingdom Ltd. The company had no input into the design, conduct, or analysis of this study and have not had advanced sight of either the data nor the manuscript. 


\section{The effect of high-speed dental handpiece coolant delivery and design on aerosol and droplet production}

Short title: Aerosol from high-speed dental handpieces

CRediT author statement:

James Allison: Conceptualization, Methodology, Formal analysis, Investigation, Data Curation, Writing - Original Draft, Writing - Review \& Editing, Visualization, Project administration, Funding Acquisition. David Edwards: Methodology, Investigation, Writing - Original Draft, Writing - Review \& Editing, Funding Acquisition. Charlotte Bowes: Methodology, Investigation, Writing - Original Draft, Writing - Review \& Editing, Funding Acquisition. Kimberley Pickering: Investigation, Writing - Review \& Editing. Christopher Dowson: Methodology, Formal analysis, Investigation, Data Curation, Writing - Review \& Editing. Simon Stone: Conceptualization, Methodology, Writing - Review \& Editing. Joanna Lumb: Conceptualization, Methodology, Writing - Review \& Editing. Justin Durham: Conceptualization, Methodology, Writing - Review \& Editing, Funding Acquisition. Nicholas Jakubovics: Methodology, Writing - Review \& Editing, Funding Acquisition. Richard Holliday: Conceptualization, Methodology, Formal analysis, Investigation, Data Curation, Writing - Original Draft, Writing - Review \& Editing, Visualization, Supervision, Project administration, Funding Acquisition. 


\title{
The effect of high-speed dental handpiece coolant delivery and design on aerosol and droplet production
}

\author{
Allison JR, Edwards D, Bowes C, Pickering K, Dowson C, Stone SJ, Lumb J, Durham J, Jakubovics N, \\ Holliday R.
}

\section{Supporting Information}

\section{Methods}

A 10-minute crown preparation of the right maxillary central incisor tooth was performed using a dental training unit (Model 4820, A-dec; OR, USA) on dental models (AG-3, Frasaco GmbH; Tettnang, Germany). Polyvinyl siloxane putty (Lab-putty, Coltene/Whaledent; Altstätten, Switzerland) was added to the mouth of the mannequin to recreate the normal dimensions of the oral cavity as described by (Dahlke et al. 2012). The procedure was performed using diamond burs in a 1:4.2 speed increasing handpiece (Ti-Max Z45L, NSK; Tochigi, Japan ) driven at $168,000 \mathrm{~min}^{-1}$ bur speed using an electric micromotor (NLX Nano, NSK; Tochigi, Japan) at $40,000 \mathrm{~min}^{-1}$ output speed. Two experiments were conducted, each in triplicate, the first with the handpiece irrigation in spray coolant configuration and the second with the handpiece in water-jet (i.e. non-chip-air) configuration (coolant flow rate of approx. $20 \mathrm{~mL} / \mathrm{min}$ ). Each procedure was performed by the same operator $(\mathrm{RH})$ without dental suction to allow examination of the relative unimpeded distribution of contamination from each instrument.

Fluorescein sodium (Sigma-Aldrich; MO, USA) was added to the irrigation reservoir of the dental unit as $2.65 \mathrm{mmol} \mathrm{L}^{-1}$ solution as a tracer to show the relative distribution of contamination from the coolant spray of both coolant configurations.

Experiments were conducted in a $308 \mathrm{~m}^{2}$ clinical simulation unit (CSU) with 6.5 air changes per hour ( $\mathrm{ACH}$; assessed by external engineering contractor) provided by a conventional hospital 
heating ventilation and air conditioning (HVAC) system. A rig with eight, $4 \mathrm{~m}$ arms was constructed around the dental unit which supported $30 \mathrm{~mm}$ diameter grade 1 qualitative filter papers (Whatman; Cytiva, MA, USA) spaced at $0.5 \mathrm{~m}$ intervals as previously described (Allison et al. 2021). During the procedure only the operator was present within the experimental area. During the collection period there were 2-3 other investigators present within the experimental area in order to collect samples. Each procedure was repeated on three separate occasions.

Contamination of filter papers was assessed as previously described (Allison et al. 2021) by eluting fluorescein from samples in distilled water, and then using a microplate reader (Synergy HT, BioTek; VT, USA) to calculate relative fluorescence units (RFU). The investigator who conducted this analysis was blind to the experimental conditions. Mean measurements of blank samples + 3 standard deviations (164 RFU) was used as the lower limit of detection; hence a zero reading was assigned to values below 164 RFU. For readings above the detection limit of the instrument (>100,000 RFU), a value of 100,000 RFU was assigned.

Data were collected using Excel (2016, Microsoft; WA, USA) and analysed using SPSS (version24, IBM Corp.; NY, USA) using basic descriptive statistics. Heatmaps were created to show distribution of contamination using Python 3 (Van Rossum \& Drake 2009). For each coordinate on the heatmap, the maximum value recorded from three repetitions of each clinical procedure was used. Logarithmic transformation was performed on the data $\left(\log _{10}\right)$.

\section{$\underline{\text { Results }}$}

Full datasets are publicly available (https://doi.org/10.25405/data.ncl.14518950). Figures S1 and S2 graphically present a summary of the distribution of contamination from the two coolant conditions. 


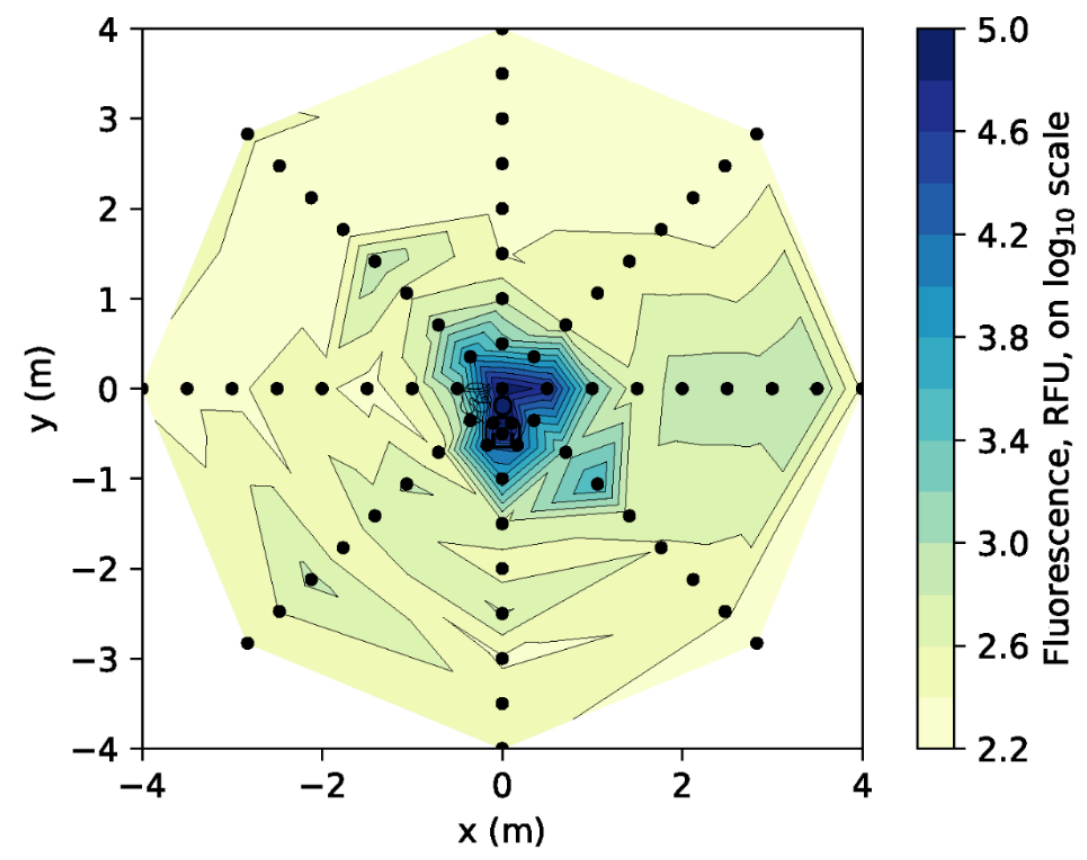

Figure S1. Heatmap presenting spectrofluorometric analysis of the samples from the anterior crown preparation using the electric micromotor handpiece with the spray mode. For each coordinate, the maximum value recorded from three repetitions of each clinical procedure was used. Logarithmic transformation was performed on the data (Log10). RFU: relative fluorescence units.
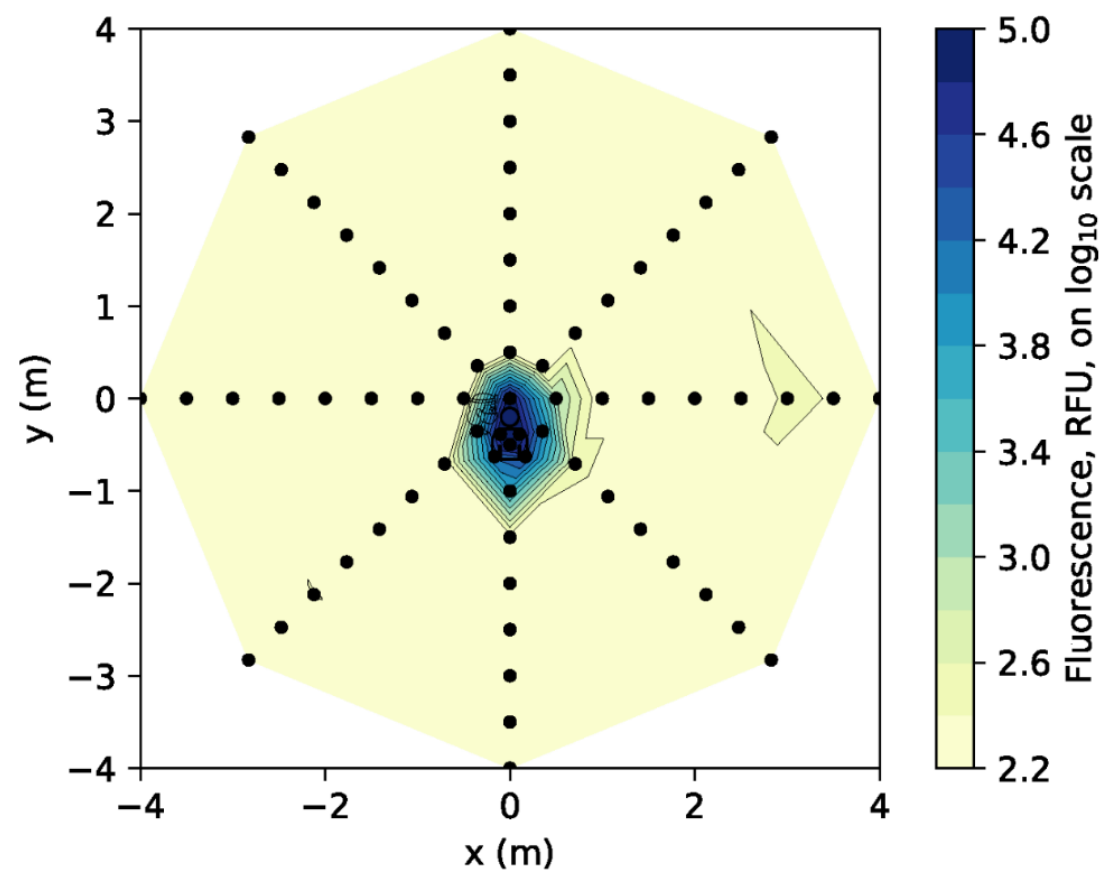

Figure S2. Heatmap presenting spectrofluorometric analysis of the samples from the anterior crown preparation using the electric micromotor handpiece with the water-jet mode. For each coordinate, the maximum value recorded from three repetitions of each clinical procedure was used. Logarithmic transformation was performed on the data (Log10). RFU: relative fluorescence units. 

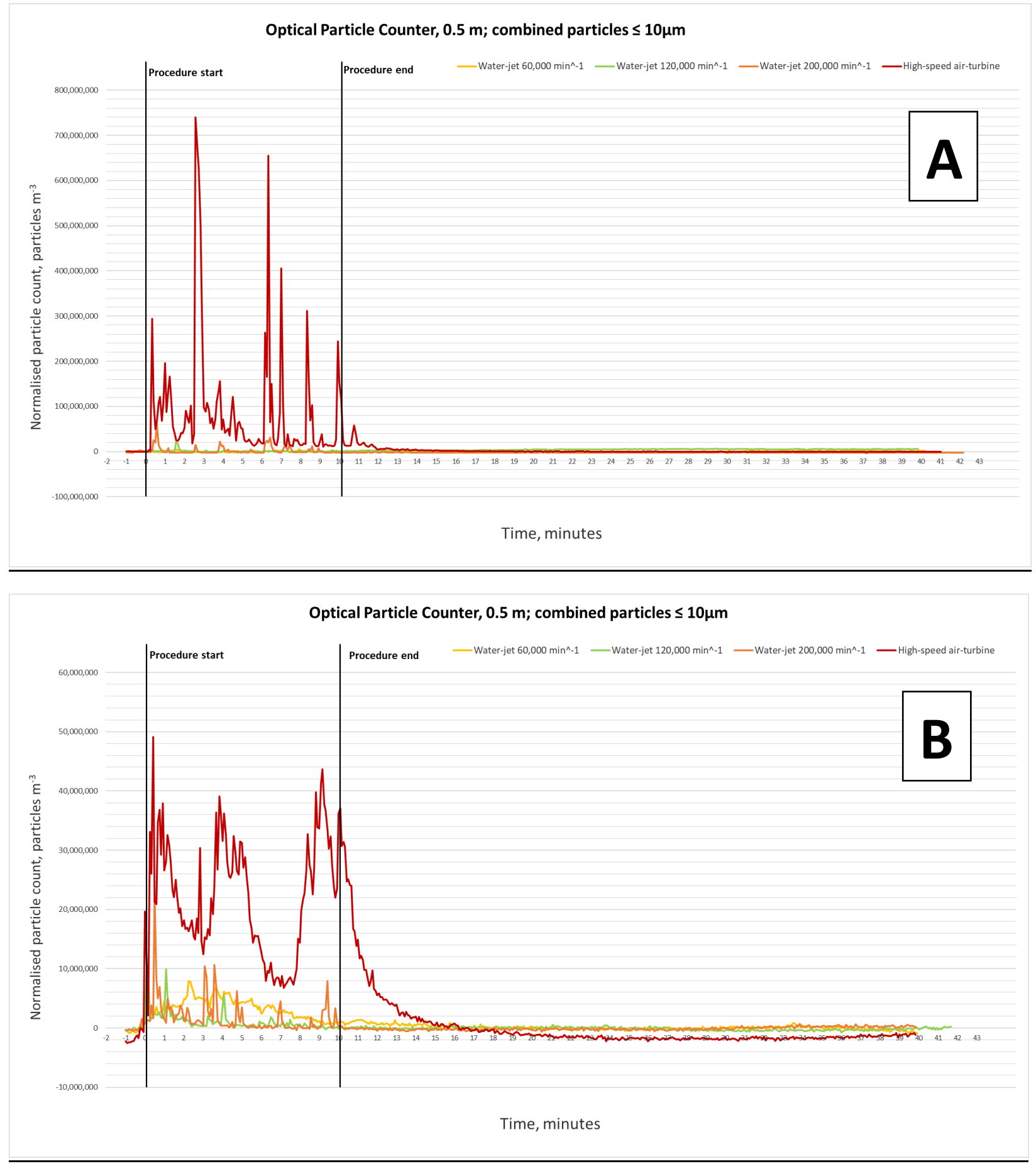

Figure S3. Suspended droplets as measured by an optical particle counter for repetitions 2 (A) and 3 (B) in addition to the repetition shown in the main paper for the $0.5 \mathrm{~m}$ location. Data were presented as normalised particle counts (particles $/ \mathrm{m}^{3}$ ) over the time-course of the experiment and total particle counts were summed across all channels. All OPC data were normalised to an internal baseline by subtracting the average counts during the 1 minute before the procedure from all particle counts. 

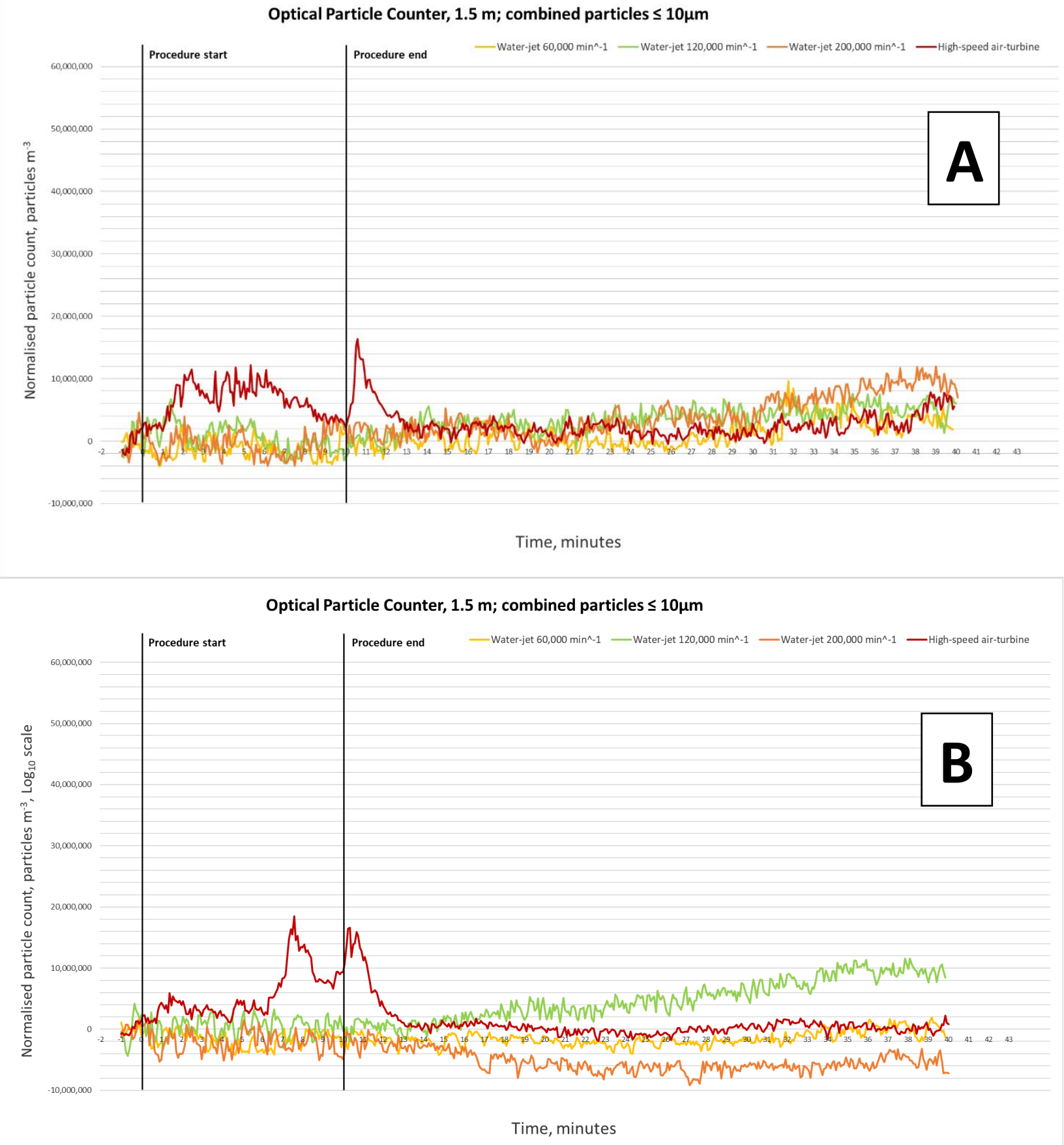

Figure S4. Suspended droplets as measured by an optical particle counter for repetitions 2 (A) and 3 (B) in addition to the repetition shown in the main paper for the $1.5 \mathrm{~m}$ location. Data were presented as normalised particle counts (particles $/ \mathrm{m}^{3}$ ) over the time-course of the experiment and total particle counts were summed across all channels. All OPC data were normalised to an internal baseline by subtracting the average counts during the 1 minute before the procedure from all particle counts. 

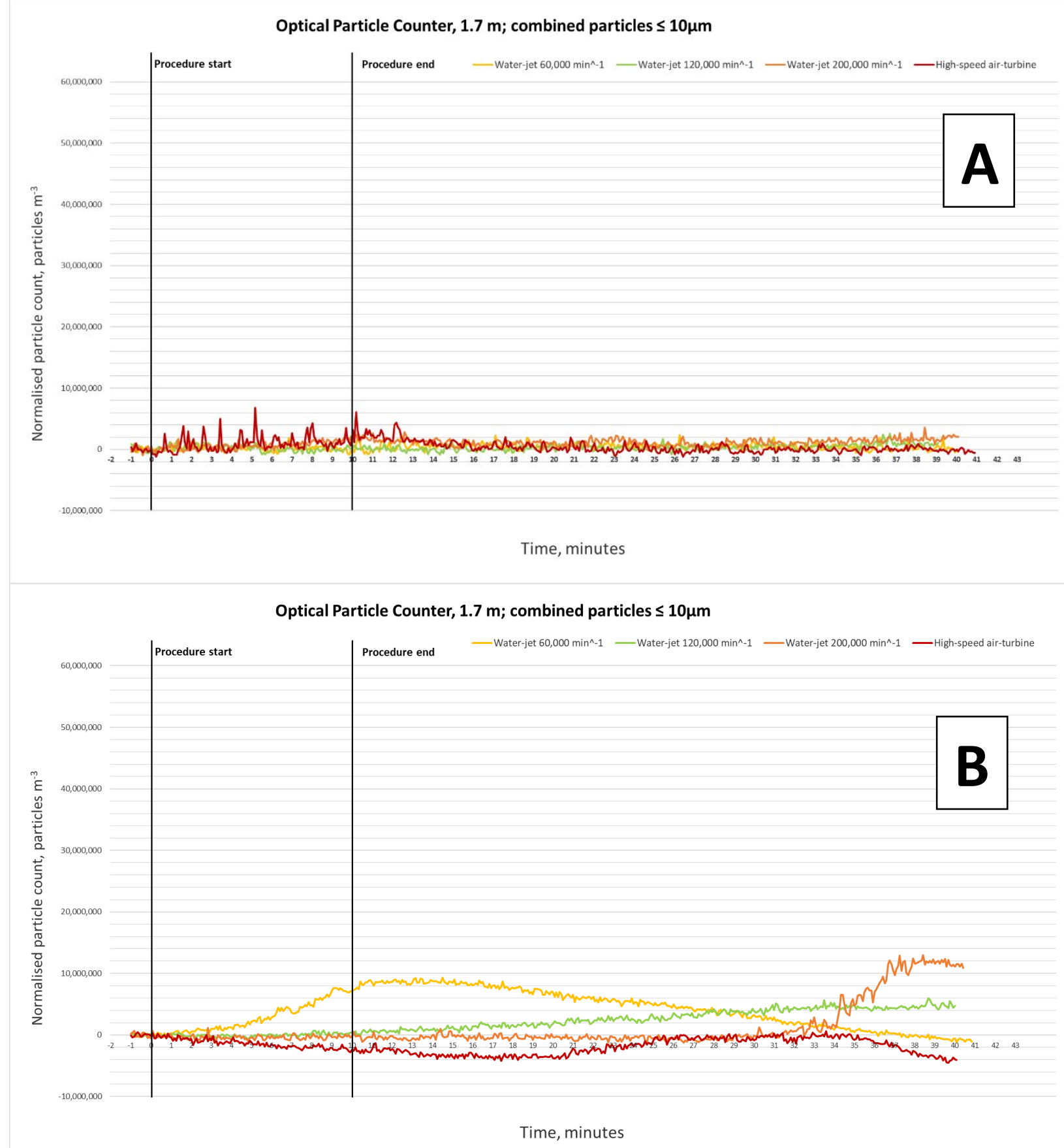

Figure S5. Suspended droplets as measured by an optical particle counter for repetitions 2 (A) and 3 (B) in addition to the repetition shown in the main paper for the $1.7 \mathrm{~m}$ location. Data were presented as normalised particle counts (particles $/ \mathrm{m}^{3}$ ) over the time-course of the experiment and total particle counts were summed across all channels. All OPC data were normalised to an internal baseline by subtracting the average counts during the 1 minute before the procedure from all particle counts. 


\section{$\underline{\text { References }}$}

Allison JR, Currie CC, Edwards DC et al. (2021) Evaluating aerosol and splatter following dental procedures: addressing new challenges for oral healthcare and rehabilitation. Journal of Oral Rehabilitation 48, 61-72.

Dahlke WO, Cottam MR, Herring MC, Leavitt JM, Ditmyer MM, Walker RS (2012) Evaluation of the spatter-reduction effectiveness of two dry-field isolation techniques. Journal of The American Dental Association 143, 1199-1204. 